Article

\title{
Two Different Scenarios for the Equilibration of Polycation-Anionic Solutions at Water-Vapor Interfaces
}

\author{
Eduardo Guzmán 1,2,*, Laura Fernández-Peña ${ }^{1}$, Andrew Akanno ${ }^{1,2}$, Sara Llamas ${ }^{1}$, \\ Francisco Ortega ${ }^{1,2}$ and Ramón G. Rubio ${ }^{1,2}$ \\ 1 Departamento de Química Física, Facultad de Ciencias, Universidad Complutense de Madrid, Ciudad \\ Universitaria s/n, 28040 Madrid, Spain \\ 2 Instituto Pluridisciplinar, Universidad Complutense de Madrid, Paseo Juan XXIII, 1, 28040 Madrid, Spain \\ * Correspondence: eduardogs@quim.ucm.es; Tel.: +34-91-394-4107
}

Received: 24 June 2019; Accepted: 11 July 2019; Published: 13 July 2019

\begin{abstract}
The assembly in solution of the cationic polymer poly(diallyldimethylammonium chloride) (PDADMAC) and two different anionic surfactants, sodium lauryl ether sulfate (SLES) and sodium $\mathrm{N}$-lauroyl-N-methyltaurate (SLMT), has been studied. Additionally, the adsorption of the formed complexes at the water-vapor interface have been measured to try to shed light on the complex physico-chemical behavior of these systems under conditions close to that used in commercial products. The results show that, independently of the type of surfactant, polyelectrolyte-surfactant interactions lead to the formation of kinetically trapped aggregates in solution. Such aggregates drive the solution to phase separation, even though the complexes should remain undercharged along the whole range of explored compositions. Despite the similarities in the bulk behavior, the equilibration of the interfacial layers formed upon adsorption of kinetically trapped aggregates at the water-vapor interface follows different mechanisms. This was pointed out by surface tension and interfacial dilational rheology measurements, which showed different equilibration mechanisms of the interfacial layer depending on the nature of the surfactant: (i) formation layers with intact aggregates in the PDADMAC-SLMT system, and (ii) dissociation and spreading of kinetically trapped aggregates after their incorporation at the fluid interface for the PDADMAC-SLES one. This evidences the critical impact of the chemical nature of the surfactant in the interfacial properties of these systems. It is expected that this work may contribute to the understanding of the complex interactions involved in this type of system to exploit its behavior for technological purposes.
\end{abstract}

Keywords: polyelectrolyte; surfactants; kinetically trapped aggregates; interfaces; surface tension; interfacial dilational rheology; adsorption

\section{Introduction}

The study of polyelectrolyte oppositely charged surfactant solutions, either in bulk or close to interfaces (fluid and solid ones), has grown very fast in the last two decades [1], mainly as result of its interest for a broad range of technological and industrial fields, e.g., drug delivery systems, food science, tertiary oil recovery, or cosmetic formulations [1-9]. Most of such applications take advantage of the chemical nature of the compounds involved, structural features of the formed complexes, and the rich phase diagrams appearing in this type of system [10-12].

Despite the extensive research, the description of the physico-chemical behavior of these colloidal systems remains controversial, in part because the self-assembly processes of polyelectrolytes and surfactants bearing opposite charges leads to the formation of non-equilibrium complexes [10,13-16]. They are expected to impact significantly on the properties of the solutions and in their adsorption at the interfaces [7]. This makes it necessary to pay attention to aspects such as the polymer-surfactant 
mixing protocol, the elapsed time from the preparation of solutions until their study, or the addition of inert electrolytes when comparisons between different studies are performed [17-19]. The role of the above-mentioned aspects in the physico-chemical properties and the phase diagrams of polyelectrolyte-surfactant solutions have been the focus of many studies, which have evidenced the complex behavior of polyelectrolyte-surfactant solutions $[6,8,17-19]$. It is worth mentioning that the non-equilibrium nature of the complexation process of polymer-surfactant solutions has an extraordinary impact on the interfacial properties of such solutions, as was recently stated by Campbell and Varga [20].

The role of the presence of non-equilibrium aggregates on the adsorption of polymer-surfactant solutions at fluid interfaces was already evidenced by the seminal works of the groups of Campbell and of Meszaros, focused on the analysis of the surface tension of polyelectrolyte-surfactant solutions [7,21-23]. However, it was necessary to use neutron reflectometry, which provides information on the composition and structure of the interfaces to deepen the most fundamental aspects of the physico-chemical behavior of these systems [24-26]. The studies of Penfold's group were a preliminary step toward the understanding of the correlations existing between the aggregation occurring in polyelectrolyte-surfactant solutions and the behavior of these complexes' fluid interfaces [27-31]. However, such works used an extended Gibbs formalism to describe the adsorption at fluid interfaces, i.e., provide a thermodynamic description. This approach was able to account for the non-regular dependences of the surface tension on the bulk concentration (surface tension peaks), even though it neglects the impact of non-equilibrium aspects [32,33]. More recently, Campbell et al. [17,18,34-39], using surface tension measurements and neutron reflectometry combined with ellipsometry, Brewster angle microscopy, and different bulk characterization techniques, tried to link the interfacial properties of the solutions to the bulk phase behavior, paying special attention to the role of the non-equilibrium effects. Their physical picture takes into account the role of the depletion of the interface as a result of the aggregation in the bulk [40], and the enrichment of the interface in virtue of direct interactions of the formed aggregates [19].

Most studies that analyze the behavior of the adsorption of polyelectrolyte-surfactant solutions at fluid interfaces only consider the interfaces as static systems. However, a comprehensive description of their behavior requires taking into consideration the response of such systems against mechanical deformations, i.e., the rheological response of the interfaces [7,41-45]. The understanding of such aspects is essential because most technological applications of interfacial systems, e.g., foam stabilization [42], rely on the response of the interfaces against mechanical perturbations [43]. The seminal studies on the rheological characterization of polyelectrolyte-surfactant layers at the water-vapor interface done by Regismond et al. [26,46] pointed out the strong synergetic effect on the interfacial properties as result of the influence of the bulk complexation process in the interfacial properties. More recent studies by Bhattacharyya et al. [47] and Monteux et al. [48] correlated the interfacial rheological response of polyelectrolyte-surfactant solutions with their ability to stabilize foams. They found that the formation of gel-like layers at the interface hindered destabilization processes such as bubble coalescence and foam drainage. Deepening the understanding of the rheological response of polyelectrolyte-surfactant solutions, Noskov et al. [26,42,43,45,49] showed that the mechanical behavior of the interface is controlled by the heterogeneity of layers, which is reminiscent of the structure of the complexes formed in solution.

It is worth mentioning that most studies in the recent literature deal with solutions containing relative low polymer concentrations, which hold limited interest from an industrial point of view. It is expected that polymer concentration can present an important contribution in both the complexation process and the interfacial properties of polyelectrolyte-surfactant solutions $[19,41,43]$. Previous studies have shown that, whereas in diluted polyelectrolyte-surfactant solutions, equilibrium between free surfactant molecules and complexes is always present in solution, the role of the free surfactant is rather limited when polymer concentration is increased. For the latter, the binding degree of surfactant molecules to the polymer chain reach values above $90 \%$, which makes it possible to assume that they are mostly complexes that are presented in solution, even for compositions in the vicinity 
of the onset of the phase separation region [50]. The differences in the complexation phenomena occurring in concentrated and diluted mixtures may significantly affect the interfacial assembly of polymer-surfactant solutions, with concentrated mixtures leading to the formation of interfacial layers, with composition mirroring the composition of the bulk solutions. The latter is far from the scenario found for diluted solutions $[50,51]$.

This work presents a comparative study of the equilibrium and dynamic properties of interfacial layers formed upon adsorption at the water-vapor interface of solution formed by poly(diallyldimethylammonium chloride) (PDADMAC) and two different anionic surfactants: sodium lauryl-ether sulfate (SLES) and sodium N-lauroyl-N-methyltaurate (SLMT). PDADMAC was chosen as the polymer because of its common utilization as a conditioner in cosmetic formulations for hair care and cleansing. Furthermore, SLES and SLMT have been recently included in formulations of shampoos to replace sodium dodecylsulfate (SDS) due to their softness and mildness, which limits skin and mucosa irritation [1].

The main aim of this work is to unravel the different interfacial behavior appearing in polycation-oppositely charged surfactant mixtures. The adsorption at the water-vapor interface is studied by surface tension measurements obtained with different tensiometers. It is worth mentioning that although polyelectrolyte-surfactant may be out of equilibrium, for simplicity we will refer to the effective property measured in this work as surface tension. In addition to the steady state measurements of the surface tension, we will follow the adsorption kinetics of the complexes at the water-vapor interface by the time evolution of the surface tension (dynamic surface tension) and the mechanical performance of the interfaces against dilation using oscillatory barrier experiments in a Langmuir trough [52]. The obtained results will be combined with the information obtained from the study of the self-assembly phenomena taking place in solution. This will provide a comprehensive description of the equilibration processes occurring during the formation of interfacial layers in this type of system. It is expected that the results contained here may help to shed light on the complex physico-chemical behavior of these systems.

\section{Materials and Methods}

\subsection{Chemicals}

PDADMAC, with an average molecular weight in the 100-200 kDa range, was purchased as a $20 \mathrm{wt}$ \% aqueous solution from Sigma-Aldrich (Saint Louis, MO, USA), and was used without further purification. SLES was supplied by Kao Chemical Europe S.L. (Barcelona, Spain) as an aqueous solution of surfactant concentration $70 \mathrm{wt} . \%$ and was purified by lyophilization followed by recrystallization of the obtained powder using acetone for HPLC (Acros Organics, Hampton, NH, USA) [50]. SLMT was synthetized and purified following the procedures described in a previous study [50]. Scheme 1 shows the molecular formula for PDADMAC and the two surfactants used in this work.

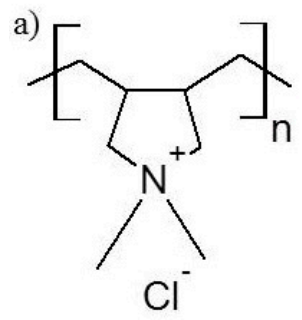

b)<smiles>CCCCCCCCCCCC(=O)N(C)CCS(=O)(=O)[OH2+]</smiles>

c)

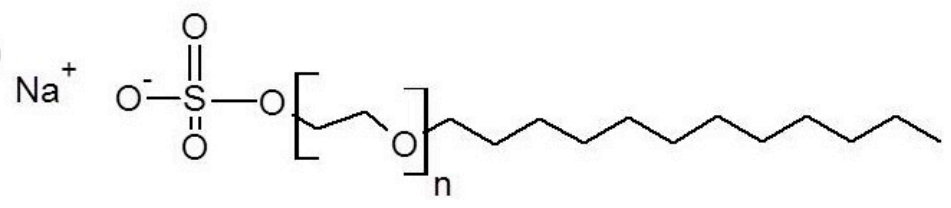

Scheme 1. Molecular formula of the three surfactants used in this work: PDADMAC (a), SLMT (b) and SLES (c). 
Ultrapure deionized water used for cleaning and solution preparation was obtained using a multicartridge purification system AquaMAX ${ }^{\mathrm{TM}}$-Ultra 370 Series. (Young Lin, Anyang, Korea). This water presents a resistivity higher than $18 \mathrm{M} \Omega \cdot \mathrm{cm}$, and a total organic content lower than $6 \mathrm{ppm}$. Glacial acetic acid and $\mathrm{KCl}$ (purity $>99.9 \%$ ) purchased from Sigma-Aldrich were used to fix the $\mathrm{pH}$ and the ionic strength of solutions, respectively.

\subsection{Preparation of Polyelectrolyte-Surfactant Solutions}

The preparation of polyelectrolyte-surfactant solutions was performed following a procedure adapted from that proposed by Llamas et al. [53]. Firstly, the required amount of PDADMAC aqueous stock solution (concentration $20 \mathrm{wt} . \%$ ) for obtaining a solution with polyelectrolyte concentration of $0.5 \mathrm{wt} . \%$ was weighted and poured into a flask. Then, $\mathrm{KCl}$ up to a final concentration of $40 \mathrm{mM}$ was added into the flask. The last step involved the addition of the surfactant and the final dilution with an acetic acid solution of $\mathrm{pH} \sim 5.6$ to reach the final composition. The addition of surfactant was performed from stock aqueous solutions ( $\mathrm{pH}$ 5.6) with a concentration one order of magnitude higher than that in the final solution. In this work, polyelectrolyte-surfactant solutions with surfactant concentration, $c_{\mathrm{s}}$, in the range $10^{-6}-10 \mathrm{mM}$ were studied. Once the solutions were prepared, these were mildly stirred (1000 rpm) for one hour using a magnetic stirrer to ensure the compositional homogenization of the solutions. Samples were left to age for 1 week prior to their use to ensure that no phase separation appeared in samples within the aging period [52].

\subsection{Techniques}

\subsubsection{Turbidity Measurements}

The turbidity of the solutions was evaluated from their transmittance at $400 \mathrm{~nm}$, obtained using a UV-Visible spectrophotometer (HP-UV 8452, Hewlett Packard, Palo Alto, CA, USA). The turbidity of the samples was determined by the optical density at $400 \mathrm{~nm}\left(\mathrm{OD}_{400}=[100-T(\%)] / 100\right.$, where $T$ is the transmittance). It is worth mentioning that neither the polyelectrolyte nor the surfactant present any absorption band above $350 \mathrm{~nm}$.

\subsubsection{Binding Isotherm}

The binding isotherm of the anionic surfactant to the polycation PDADMAC was determined by potentiometric titration using a surfactant selective electrode model 6.0507.120 from Metrohm (Herisau, Switzerland). The binding degree of surfactant $\beta$ was estimated from the potentiometric measurements, as was proposed by Mezei and Meszaros [22]

$$
\beta=\frac{c_{s}^{\text {free }}}{c_{\text {monomer }}}
$$

where $c_{s}^{\text {free }}$ and $c_{\text {monomer }}$ are the concentrations of free surfactant in solution and charged monomers of the polyelectrolyte chains, respectively. This method of determining the binding isotherm provides information about the amount of free surfactant remaining in the solution.

\subsubsection{Surface Tension Measurements}

Surface tension measurements as functions of the surfactant concentration (SLMT or SLES) for pure surfactant and polyelectrolyte-surfactant solutions were performed using different tensiometers. In all the cases, the adsorption was measured until the steady state conditions were reached. Special care was taken to limit the evaporation effects. Each value was obtained as an average of three independent measurements. All experiments were performed at $25.0 \pm 0.1^{\circ} \mathrm{C}$. From the results of the experiments, it is possible to define the surface pressure as $\Pi\left(c_{\mathrm{s}}\right)=\gamma_{0}-\gamma\left(c_{\mathrm{s}}\right)$, where $\gamma_{0}$ is the surface 
tension of the bare water-vapor interface and $\gamma\left(c_{\mathrm{s}}\right)$ is the surface tension of the solution-vapor interface. Further details on surface tension experiments can be obtained from a previous study [23].

- Surface force tensiometers. Two different surface force tensiometers were used to measure the equilibrium surface tension: a surface force balance from Nima Technology (Coventry, UK), fitted with a disposable paper plate (Whatman CHR1 chromatography paper) as a contact probe; and a surface force tensiometer Krüss K10 (Hamburg, Germany), using a Pt Wilhelmy plate as a probe.

- Drop profile analysis tensiometer. A home-built drop profile analysis tensiometer in pendant drop configuration allowed determination of the surface tension of the water-vapor interface. This tensiometer enabled evaluation of the time dependence of the surface tension during the adsorption process, thus providing information related to the adsorption kinetics.

\subsubsection{Dilational Rheology}

A Nima 702 Langmuir balance from Nima Technology equipped with a surface force tensiometer was used to measure the response of the surface tension against sinusoidal changes in the surface area. Thus, it is possible to obtain information about the dilational viscoelatic moduli of the water-vapor interface $\varepsilon^{*}=\varepsilon^{\prime}+i \varepsilon^{\prime \prime}$, with $\varepsilon^{\prime}$ and $\varepsilon^{\prime \prime}$ being the dilational elastic and viscous moduli, respectively, in the frequency range of $10^{-1}-10^{-2} \mathrm{~Hz}$ and at an area deformation amplitude $\Delta u=0.1$, which was verified to be an appropriate value to ensure results within the linear regime of the layer response [52].

\section{Results and Discussion}

\subsection{PDADMAC-Surfactants Assembly in Solution}

The equilibrium condition implies that the chemical potential of all the species in both the bulk and at the interfaces are the same. Therefore, any physical understanding of the latter implies knowledge of the behavior of the different species in the bulk. Figure 1a shows the surfactant-binding isotherms deduced from electromotive force (EMF) measurements. Comparing the curves of EMF obtained for surfactants and PDADMAC-surfactant solutions, it is possible to obtain the binding isotherms for the corresponding surfactant to PDADMAC chains following the approach described by Mezei and Meszaros [50]. The results point out a high degree of binding over the whole range of studied compositions, providing an additional confirmation of the high efficiency of PDADMAC in binding anionic surfactants. Campbell et al. [38] found for PDADMAC-SDS solutions binding degrees of surfactant to PDADMAC close to 0.3 in the vicinity of the isoelectric point (surfactant concentration around $0.2 \mathrm{mM}$ ). The extrapolation of such results in similar conditions to those considered in this work, i.e., polymer concentration 50-fold the one used by Campbell et al. [38,52], and assuming that the binding is not significantly modified either for the surfactant structure or for the differences in the ionic strength, takes the binding degree at charge neutralization to a value $<1 \%$. This is just the situation found here, where binding isotherms evidence that the amount of free surfactant in solution remains below $10 \%$, even for the highest surfactant concentrations. The low concentration of free surfactant in solution allows us to assume hereinafter that the bulk has a negligible free-surfactant concentration.

Figure $1 \mathrm{~b}$ shows the dependence of the optical density of the samples on the surfactant concentration for the solutions of PDADMAC and the two surfactants. Similar qualitative concentration dependences of the optical density were found for both polyelectrolyte-surfactant systems. It may safely be expected that all of the studied compositions for PDADMAC-surfactant solutions fall in an equilibrium one-phase region, showing optically transparent solutions. This comes from the fact that the number of surfactant molecules available in solution is not high enough to neutralize the charge of all the monomers in the polyelectrolyte chains, thus leading to the formation of undercompensated cationic complexes in solution. Indeed, considering the high polymer concentration, simple calculations suggest the existence of around 36 monomers for each surfactant molecule for a surfactant concentration of approximately $1 \mathrm{mM}$. Therefore, assuming the complete binding of surfactant molecules to the polymer chains, around 35 monomers remain positively charged in the complexes, supporting the 
formation of transparent samples within the entire concentration range. However, contrary to what was expected for solutions with compositions far from the neutralization, the solutions formed by undercompensated complexes show an increase of the turbidity for the highest surfactant concentration. Therefore, for such concentrated solutions, the system should get close to the onset of where the two phase region occurs, even though no signature of charge neutralization was found from electrophoretic mobility measurements. This results from the mixing protocol used for solution preparation, which proceeds during the initial step by mixing a concentrated polymer solution with a concentrated surfactant solution. This precursor solution is them diluted up to the stated bulk composition. It may be expected that this methodology leads, due to the Marangoni stress created, to the formation of persistent kinetically-trapped aggregates that persist even upon dilution, leading to the appearance of a two-phase system far off the real neutralization point of the system [7,54]. These results contrast with those reported in other mixtures studied in the literature. In such systems, the increase on the optical density of the samples results from the formation of charge compensated complexes. The last is associated with the transition from a composition region, in which the charge of the complexes is governed by the excess of charged monomers to another region, in which the excess of bound surfactant to the polymer chain controls the charge of the formed complexes, i.e., a charge inversion transition $[20,38,55]$. The above results show that the production of kinetically-trapped aggregates during mixing can lead to turbid mixtures far from the real equilibrium phase separation [3]. Preliminary results have shown that the above discussed scenario changes significantly when the interaction of PDADMAC with betaine derived surfactants is considered. In such systems, even the polyelectrolyte-surfactant interactions occur through the negatively charged group in the terminal region of the polar head, the formation of kinetically-trapped aggregates is hindered, probably as a result of the electrostatic repulsion associated with the positively charged groups in the zwitterionic surfactant [56].
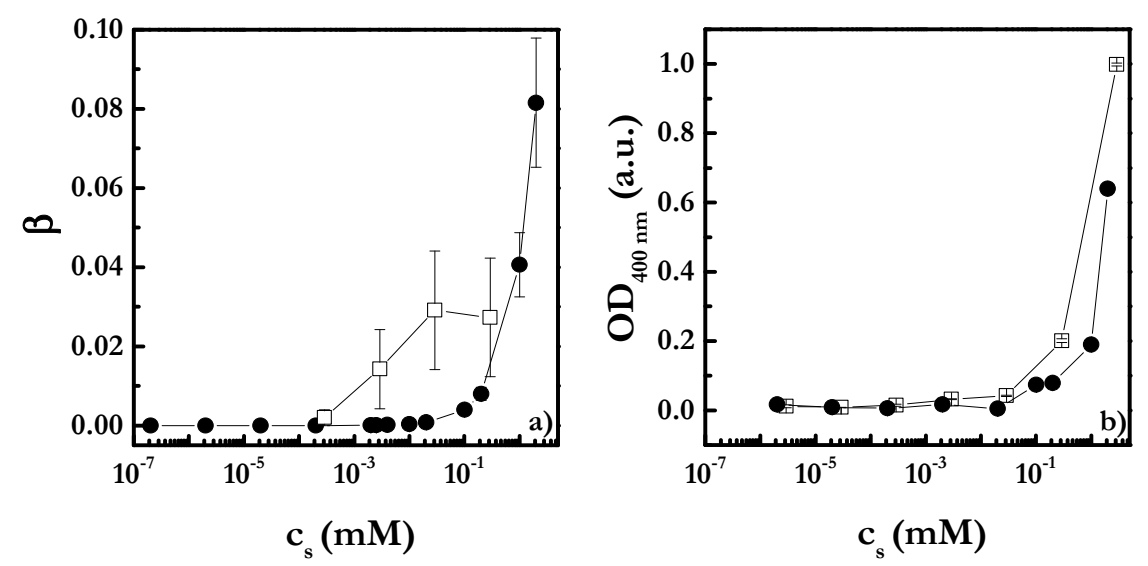

Figure 1. (a) Binding isotherms for surfactants on PDADMAC as a function of the initial concentration of surfactant in bulk. (b) Surfactant concentration dependences of the optical density of the solution, measured at $400 \mathrm{~nm}$. Note: $(\square)=$ PDADMAC-SLMT; $(\bullet)$ = PDADMAC-SLES solutions. Lines are guides for the eyes. The results correspond to PDADMAC-surfactant mixtures containing a fixed PDADMAC concentration of $0.5 \mathrm{wt} \%$, and left to age for one week prior to measurement.

\subsection{Equilibrium Adsorption at the Water-Vapor Interface}

The evaluation of the surface pressure of solutions containing surface active compounds helps to understand the mechanisms involved in the equilibration of the water-vapor interface. Figure 2a shows the surface pressure dependences on the surfactant concentrations and on the PDADMAC concentration for the adsorption of the two surfactants and the polymer at the water-vapor interface (note that all solutions were prepared with the same $\mathrm{pH}$ and inert salt concentration as the polyelectrolyte-surfactant solutions). The results show that the surface activity of PDADMAC is negligible, at least up to concentrations that are 20-fold the one used in our work. This is in good agreement with the previous 
study by Noskov et al. [57] and with the negligible surface excess found for PDADMAC using neutron reflectrometry [38].
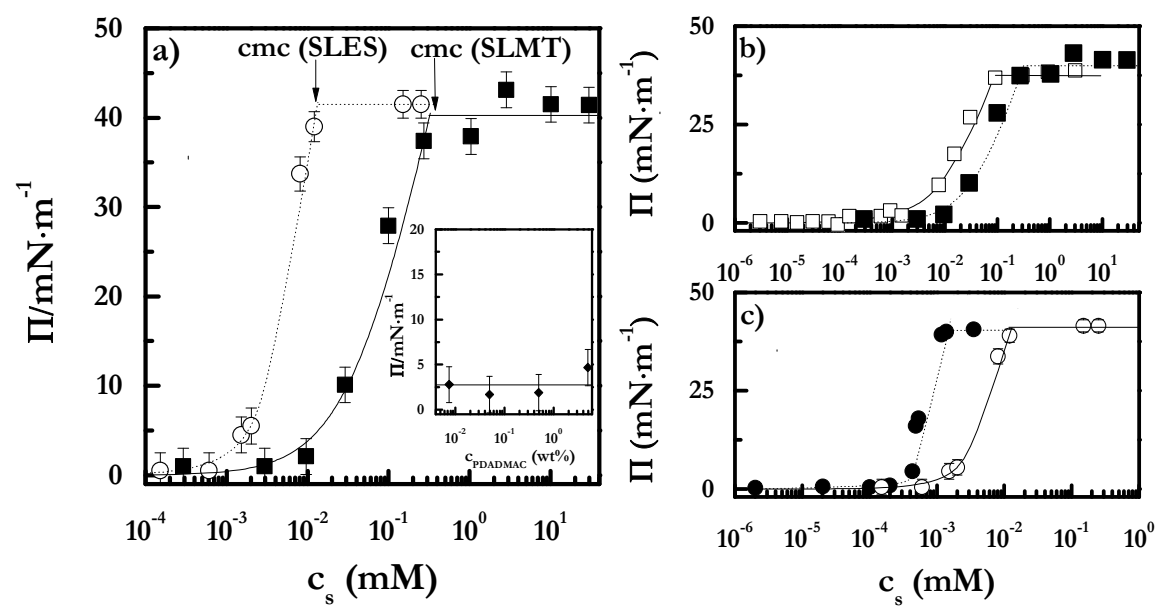

Figure 2. Results obtained using a drop profile analysis tensiometer: (a) Surface pressure dependence on surfactant concentration for the adsorption of pure SLES $(\bigcirc)$ and SLMT $(\boldsymbol{\square})$ at the water-vapor interface; $\mathrm{cmc}$ for both surfactants is marked. The inserted panel represents the surface pressure dependence on PDADMAC concentration for the adsorption of pure PDADMAC at the water-vapor interface. (b) Surface pressure dependence of SLMT concentration for pure SLMT ( $\mathbf{a}$ ) and PDADMAC-SLMT (口) solutions. (c) Surface pressure dependence of SLES concentration for pure SLES $(\bigcirc)$ and PDADMAC-SLES $(\bullet)$ solutions. The lines are guides for the eyes. The results for PDADMAC-surfactant mixtures correspond to mixtures containing a fixed PDADMAC concentration of $0.5 \mathrm{wt} . \%$, and left to age for one week prior to measurement.

The adsorption behavior of SLMT and SLES is the expected for typical ionic surfactants. The $\Pi$ increases with the bulk concentration up to the point that the surfactant concentration overcomes the threshold defined by the critical micellar concentration ( $\mathrm{cmc}$ ). Afterwards, $\Pi$ remains constant with further increases of surfactant concentration. It is worth mentioning that the results obtained using different tensiometers (surface force tensiometer with Pt Wilhelmy as a probe plate and drop profile analysis tensiometer) agree within the combined error bars for the adsorption of both surfactants at the water-vapor interface. The surface pressure isotherms allow one to estimate the $\mathrm{cmc}$ of the pure surfactants, which showed values of around $10^{-2}$ and $10^{-1} \mathrm{mM}$ for SLES and SLMT, respectively.

The comparison of the results obtained for the adsorption of pure surfactants at the water-vapor interface with those obtained for the adsorption of PDADMAC-surfactant solutions shows that for the lowest surfactant concentrations the surface pressure values are similar for pure surfactant and polyelectrolyte-surfactant solutions. This is the result of the low coverage of the interface (see Figure $2 b, c)$. In such conditions, the surface excess is not high enough to produce any significant change in the surface free energy, and hence the $\Pi$ values remain close to those of the bare water-vapor interface. The increase of the surfactant concentration leads to the increase of $\Pi$ for both surfactant and polyelectrolyte-surfactant solutions. This increase starts for surfactant concentrations around one order of magnitude lower when polyelectrolyte-surfactant solutions are considered, which is a signature of the existence of a synergetic effect for the increase of the surface pressure as a result of the interaction in the solution of the polyelectrolyte and the surfactant. This is in agreement with previous results reported in the literature for several polyelectrolyte-surfactant systems $[3,31,50,58]$. The above-mentioned synergetic effects do not influence the adsorption behavior of solutions formed by PDADMAC and zwitterionic surfactants derived from the betaines, as was shown in preliminary results. This could be ascribed to the aforementioned differences in the aggregation process occurring in the bulk [56]. 
The study of the surface tension isotherms obtained for polymer-surfactant mixtures using different tensiometric techniques can help to understand the complexity of the interfacial behavior appearing when faced with these systems. Figure $3 a, b$ shows that the surface tension isotherms obtained using different tensiometers reveal different features for PDADMAC-SLMT and PDADMAC-SLES solutions. PDADMAC-SLES solutions show similar surface pressure isotherms within the combined error bars, independent of the tensiometer used, and no evidences of the appearance of non-regular trends, either as surface tension peaks [38] or surface tension fluctuations [7], on the dependence of the surface pressure with the surfactant concentration were found. This contrasts with the results obtained for PDADMAC-SLMT solutions, in which the use of a surface force tensiometer with a Pt Wilhelmy plate as probe led to results that were significantly different to those obtained using the other tensiometers. The existence of such differences was previously reported in a study by Noskov et al. [31].

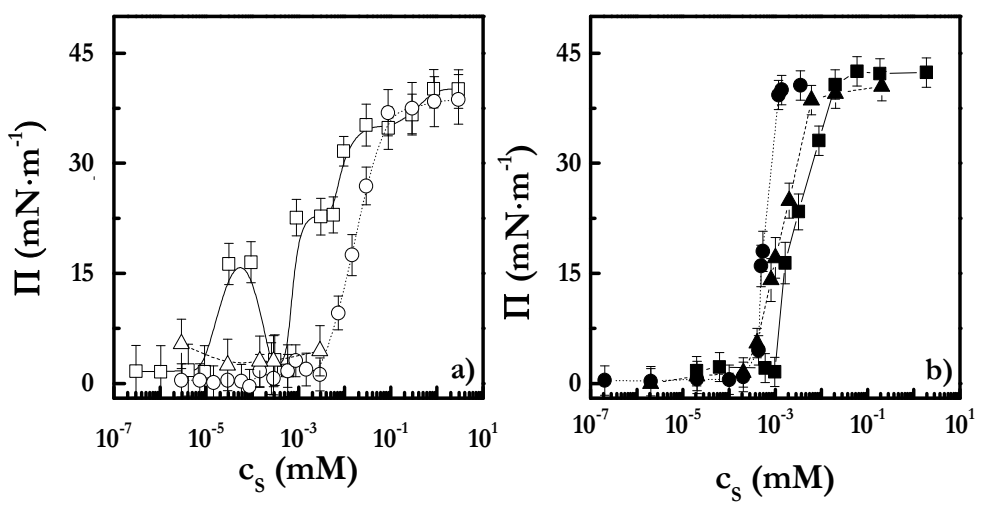

Figure 3. Surface pressure isotherms for solutions of PDADMAC with the two surfactants, obtained using different tensiometers. (a) Isotherms for PDADMAC-SLMT solutions. (b) Isotherms for PDADMAC-SLES solutions. Note: ( $\square$ and $\mathbf{\square})$ Surface force tensiometer with Pt Wilhelmy plate as contact probe; ( $\bigcirc$ and $\bullet$ ) surface force tensiometer with paper Wilhelmy plate as contact probe; $(\Delta$ and $\mathbf{\Delta})$ drop profile analysis tensiometer. The lines are guides for the eyes. The results correspond to PDADMAC-surfactant mixtures containing a fixed PDADMAC concentration of $0.5 \mathrm{wt} . \%$ left to age for one week prior to measurement.

The differences found in the tensiometric behavior of PDADMAC-SLES and PDADMAC-SLMT solutions are correlated to differences in the equilibration mechanism of the interface. Assuming that the assembly of the polyelectrolyte-surfactant in solutions leads to the formation of kinetically trapped aggregates in both cases, this can evolve following different mechanisms upon adsorption at fluid interfaces. For PDADMAC-SLMT solutions, the appearance of surface tension fluctuations far from the phase separation region may be associated with the fact that upon adsorption at the water-vapor interface of the kinetically trapped aggregates can remain as isolated aggregates embedded at the interface. These do not dissociate spontaneously to form a kinetically trapped film at the interface. As a consequence, the trapped aggregates may adsorb onto the rough surface of the Pt Wilhelmy plate, changing its contact angle, which results in non-reliable surface tension values for the considered aggregates. This scenario is in agreement with the neutron reflectometry results obtained by Llamas et al. [50]. Their results showed a monotonic increase of the surface excess at the interface with the surfactant concentration, confirming that the surface tension fluctuations do not result from fluctuations of the interface composition. The behavior changes significantly when the adsorption of PDADMAC-SLES solutions is considered. In this case, the absence of surface tension fluctuation or significant differences in the results obtained using different tensiometers suggests the existence of dissociation and spreading of the kinetically trapped aggregates upon adsorption at the interface. Thus, the equilibration of the interface after the adsorption of the kinetically trapped aggregates occurs because of its dissociation, which is followed by the spreading of the complexes across the interface as a result of Marangoni flow associated with the lateral heterogeneity of the interface $[38,42,50,59]$. 
The differences in the adsorption mechanisms of PDADMAC-SLES and PDADMAC-SLMT complexes at the water-vapor interface may be explained on the bases of the molecular structures of the surfactant and the possibility to establish a cohesion interaction with the surrounding media. SLMT presents a hydrophobic tail formed by an alkyl chain, which tends to minimize the number of contact points with water, which favors the formed aggregates remaining as compact aggregates at the water-vapor interface upon adsorption. On the contrary, the presence of oxyethylene groups in SLES makes the dissociation and spreading of the complexes easier as a result of the possible formation of hydrogen bonds of the surfactant molecules with water. Surprisingly, studies on the adsorption of PDADMAC-SLES and PDADMAC-SLMT mixtures onto solid surfaces have evidenced a scenario compatible with that described for the adsorption at the fluid interfaces, where PDADMAC-SLES films present a topography reminiscent of the formation of extended complexes attached to the interface, whereas PDADMAC-SLMT films present a higher lateral heterogeneity [51,60]. Further confirmation of the discussed mechanisms may be obtained from the analysis of the adsorption kinetics at the water-vapor interface of the polyelectrolyte-surfactant solutions.

\subsection{Adsorption Kinetics at the Water-Vapor Interface}

The analysis of the adsorption kinetics of polymer-surfactants at the water-vapor interface is a powerful tool for deepening the understanding of the mechanistic aspects of the adsorption of complexes. This is done by studying the time evolution of the surface pressure (dynamic surface pressure) during the adsorption process. The adsorption kinetics have been measured using a drop shape analysis tensiometer. As expected, the adsorption of polymer-surfactant solutions at fluid interfaces is slower than that corresponding to pure surfactant $[16,50]$. Figure 4 shows the dynamics surface pressure obtained for the adsorption of PDADMAC-SLMT and PDADMAC-SLES solutions at the water-vapor interface.

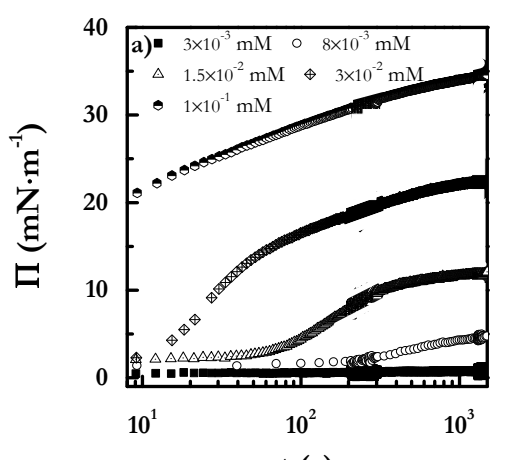

t (s)

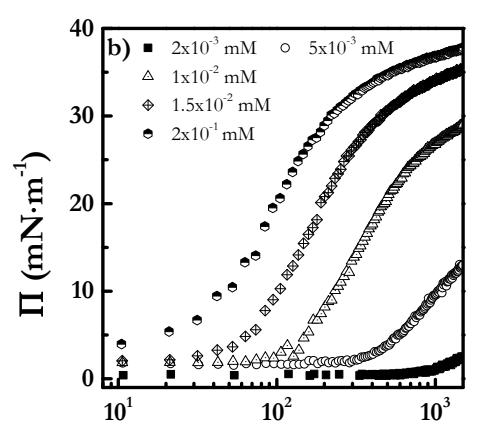

t (s)

Figure 4. (a) Dynamic surface pressure for PDADMAC-SLMT solutions with different surfactant concentrations. (b) Dynamic surface pressure for PDADMAC-SLES solutions with different surfactant concentrations. The results correspond to PDADMAC-surfactant mixtures containing a fixed PDADMAC concentration of $0.5 \mathrm{wt} . \%$, and left to age for one week prior to measurement.

The analysis of the adsorption kinetics show clearly that the increase of the surfactant concentration leads to the faster increase of the surface pressure, due to the higher hydrophobicity of the formed complexes. A more detailed analysis points out that whereas the adsorption of PDADMAC-SLMT is characterized by the monotonous increase of the surface pressure with time over the whole concentration range, the adsorption of PDADMAC-SLES presents an induction time that is reduced as the SLES concentration increases. Such differences are due to the differences in the processes involved in the equilibration of the interface. 
The induction time in the adsorption of PDADMAC-SLES is explained considering that the equilibration of the interface proceeds following a two-step mechanism, as occurs for protein adsorption at fluid interfaces [61]. Firstly, polymer-surfactant complexes attach to the water-vapor interface as kinetically trapped aggregates until the surface excess overcomes a threshold value, after which point the adsorbed complexes undergo a dissociation and spreading process, which is responsible for the surface pressure increase [41,59]. It is worth mentioning that the decrease of the induction time with the increase of surfactant concentration results from the faster saturation of the interface, i.e., the shortening of the time needed to overcome the surface excess threshold, which leads to a prior surface pressure rise. The scenario found for PDADMAC-SLMT solutions is different to that described for PDADMAC-SLES, and the absence of the induction time is a signature of a difference in the equilibration mechanism of the interfacial layer. For PDADMAC-SLMT, the increase of the surface pressure is associated with the adsorption of isolated kinetically trapped aggregates that coalesce as the surfactant concentration increases. In this case, the adsorbed complexes remain compact without any significant dissociation. The above discussed results point out the existence of differences in the mechanisms for the equilibration of the interface of the polycation-anionic surfactant solution as result of the differences in the type of surfactant. The first one involves the dissociation and spreading of the pre-adsorbed kinetically trapped aggregates (PDADMAC-SLES), whereas the second one relies directly on the saturation of the interface with kinetically trapped aggregates. This proves that the adsorption of PDADMAC-SLMT leads to appreciable modifications of the surface pressure for surfactant concentrations one order of magnitude higher than PDADMAC-SLES as a result of the negligible effect of the isolated aggregates over the surface pressure of the bare water-vapor interface until their concentration is high enough. On the contrary, for PDADMAC-SLES, the dissociation and spreading of the aggregates enables the distribution of surface active material along the whole interface, and consequently the surface pressure starts to increase for lower surfactant concentrations as a result of the formation of interfacial layers in which complexes are extended along the interface.

\subsection{Interfacial Dilational Rheology}

The above discussion was devoted to the study of the adsorption at interfaces with fixed surface areas. However, from a technological point of view, the understanding of the response of the interface against external mechanical perturbations is essential because this provides important insights into the relaxation processes involved in the equilibration of interfacial layers $[25,48,62,63]$. The dependences of the dilational viscoelastic moduli ( $\varepsilon^{\prime}$ represents the dilational elastic modulus and $\varepsilon^{\prime \prime}$ the viscous modulus) on the surfactant concentration and the deformation frequency provide complementary information for the better understanding of the complexity of the mechanism involved in the equilibration of the interfaces, helping to give a more detailed picture of the physical processes governing the formation of adsorption layers from polymer-surfactant solutions [64]. It must be stressed that for both PDADMAC-SLMT and PDADMAC-SLES solutions, the values of $\varepsilon^{\prime \prime}$ are negligible in relation to those of $\varepsilon^{\prime}$, with the ratio $\varepsilon^{\prime \prime} / \varepsilon^{\prime}$ decreasing as the surfactant concentration increases. Hence, for the sake of simplicity only the behavior of $\varepsilon^{\prime}$ will be discussed. Figure 5 shows the concentration dependences of the elastic modulus for PDADMAC-SLMT and PDADMAC-SLES layers.

The results indicate that the dependence of $\varepsilon^{\prime}$ on the frequency is expected for the formation of layers at fluid interfaces, with $\varepsilon^{\prime}$ increasing with the deformation frequency. Furthermore, the concentration dependence of $\varepsilon^{\prime}$ is similar to that found for layers of surface active materials at fluid interfaces [46], with $\varepsilon^{\prime}$ increasing with the surfactant concentration from the value corresponding to the clean interface, reaching a maximum and then dropping down again to quasi-null values for the highest surfactant concentrations. A careful examination of the values obtained for the elasticity modulus for each system indicate that PDADMAC-SLES layers present values that are more than twice those obtained for PDADMAC-SLMT solutions independent of the considered frequency. This is again indicative of the different features of the interfacial layers. For PDADMAC-SLES layers, the spreading of material along the interface leads to the formation of extended complexes that can build a cross-linked network, 
increasing the elastic modulus of the interfacial layers. This cross-linking process is not possible when the interfacial layer is formed by compact kinetically trapped aggregates, as in PDADMAC-SLMT layers, leading to lower values of the elastic modulus of the interface.
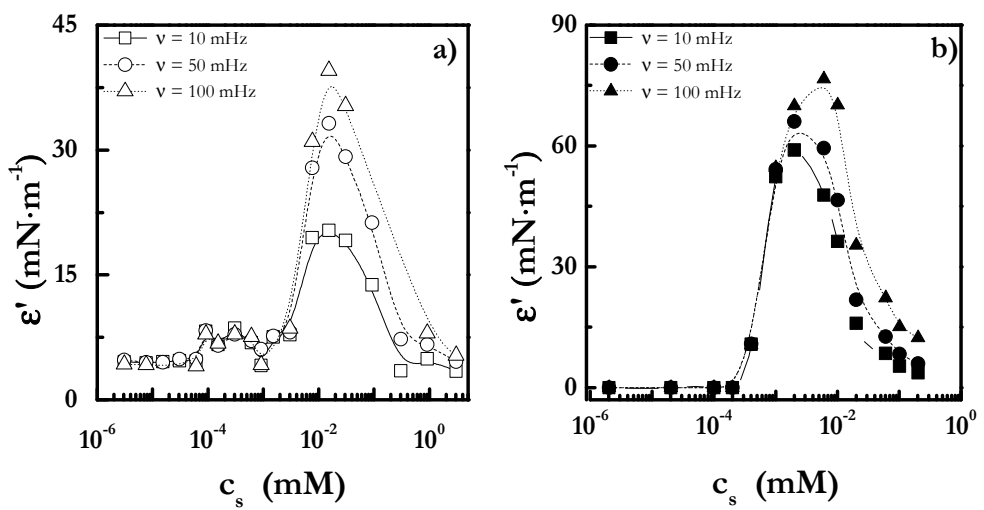

Figure 5. (a) Concentration dependences of the elastic modulus for PDADMAC-SLMT adsorption layers as were obtained from oscillatory barrier experiments performed at different frequencies. (b) Concentration dependences of the elastic modulus for PDADMAC-SLES adsorption layers, obtained from oscillatory barrier experiments performed at different frequencies. Note: ( $\square$ and $\boldsymbol{\square}) v=0.01 \mathrm{~Hz}$; $(\bigcirc$ and $\bullet$ ) $v=0.05 \mathrm{~Hz}$; $(\Delta$ and $\boldsymbol{\Delta}) v=0.10 \mathrm{~Hz}$. The lines are guides for the eyes. For the sake of clarity, only results corresponding to some of the explored frequencies $(v)$ are shown, with the other frequencies presenting similar dependences. The results correspond to PDADMAC-surfactant mixtures containing a fixed PDADMAC concentration of $0.5 \mathrm{wt} . \%$, and left to age for one week prior to measurement.

The frequency dependences of the elasticity modulus can be described in terms of the rheological model proposed by Ravera et al. [64,65] (see Figure 6a for an example). According to this model the frequency dependence of the viscoelastic modulus accounts for the initial adsorption of the polymer-surfactant complexes at the water-vapor interface as a diffusion-controlled process that is coupled to a second step associated with the internal reorganization of the adsorbed layers. Thus, taking into account the aforementioned framework, it is possible to describe the complex viscoelastic modulus with the following expression:

$$
\varepsilon^{*}=\frac{1+\xi+i \xi}{1+2 \xi+2 \xi^{2}}\left[\varepsilon_{0}+\left(\varepsilon_{1}-\varepsilon_{0}\right) \frac{1+i \lambda}{1+\lambda^{2}}\right]
$$

where $\xi=\sqrt{v_{D} / v}$, with $v_{\mathrm{D}}$ and $v$ being the characteristic frequency of the diffusion exchange and the frequency of deformation, respectively, and $\lambda=v_{1} / v$, with $v_{1}$ being the characteristic frequency of the extra relaxation process. Additionally, $\varepsilon_{0}$ and $\varepsilon_{1}$ represent the Gibbs elasticity and the high frequency limit elasticity within the frequency range considered, respectively. The validity of the discussed model, beyond confirming the complexity of the mechanisms involved in the equilibration of the interfacial layers formed by polyelectrolyte-surfactant solution, provides a description of the processes involved. It is expected that the equilibration of the interfacial occurs in the first stage by the diffusion-controlled adsorption of the kinetically trapped aggregates, and then such complexes undergo different reorganization processes depending on their nature. The existence of a two-step mechanism is in agreement with the picture proposed by Noskov et al. [45] for the equilibration of adsorption layers of PDADMAC-SDS at the water-vapor interface.

Figure $6 \mathrm{~b}, \mathrm{c}$ show the concentration dependences for the characteristic frequencies of the two dynamic processes appearing for the interfacial layers. As may be expected considering the different nature of the dynamic processes involved in the equilibration of the interfacial layer, $v_{1}$, which is the frequency corresponding to the interfacial relaxation process, presents higher values than those associated with the diffusional transport, $v_{\mathrm{D}}$, for both PDADMAC-SLMT and PDADMAC-SLES solutions. This behavior can be explained by assuming that the interfacial relaxation process, involving 
the reorganization of materials at the interface, occurs only when a certain degree of material is adsorbed at the interface.

The results show that both $v_{\mathrm{D}}$ and $v_{1}$ increase in concentration for both studied systems. This increase can be explained in the case of $v_{D}$ as a result of the enhanced surface activity of the kinetically trapped aggregates, as the surfactant concentration increases due to their higher hydrophobicity. Furthermore, the values of $v_{\mathrm{D}}$ are in a similar range for PDADMAC-SLMT and PDADMAC-SLES, which is in agreement with the similar origin of the process in both systems and the similarities of the complexes formed according to the above discussion. The slightly smaller values of $v_{\mathrm{D}}$ found for PDADMAC-SLMT than for PDADMAC-SLES may result from different sizes of the complexes formed in the solution. The dependence of $v_{1}$ is assumed to be because of the increase of surfactant in solution leading to an increase of the surface excess of complexes at the interface, which facilitates their reorganization within the interface. The higher values of $v_{1}$ for PDADMAC-SLMT solution than PDADMAC-SLES solutions, at almost one order of magnitude, are ascribable again to the differences in the structure of the interfacial layers. Thus, the diffusion of extended complexes within the interface can occur across longer distances within the interface than that of compact aggregates, and consequently this process involves longer time scales.
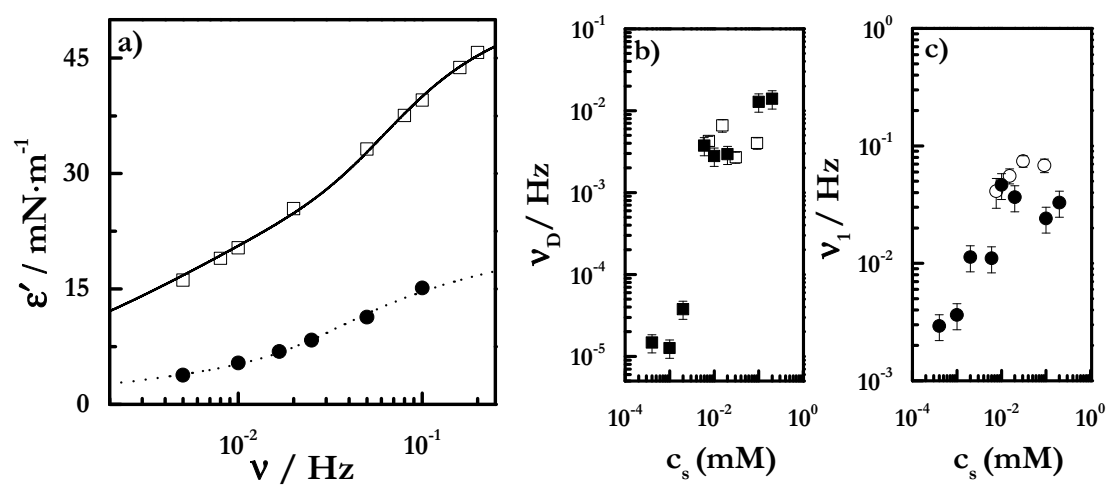

Figure 6. (a) Examples of frequency dependences of the elastic modulus for interfacial layers of PDADMAC-SLMT ( $\square)$ and PDADMAC-SLES $(\bullet)$ and for solutions with surfactant concentration of $0.1 \mathrm{mM}$. Symbols represent the experimental data and the lines are the theoretical curves obtained from the analysis of the experimental results in term of the theoretical model described by Equation (2). (b) Concentration dependences of $v_{\mathrm{D}}$ for PDADMAC-SLMT ( $\square$ ) and PDADMAC-SLES ( $\left.\mathbf{\square}\right)$. (c) Concentration dependences of $v_{1}$ for PDADMAC-SLMT $(\bigcirc)$ and PDADMAC-SLES $(\bullet)$. $(\mathbf{b}, \mathbf{c})$ Symbols represent the experimental data and the lines are guides for the eyes. The results correspond to PDADMAC-surfactant mixtures containing a fixed PDADMAC concentration of $0.5 \mathrm{wt} . \%$, and left to age for one week prior to measurement.

\section{Conclusions}

The mechanisms involved in the equilibration of interfacial layers formed by adsorption of PDADMAC and two different anionic surfactants (SLMT and SLES) have been studied by surface tension (equilibrium and dynamics) and interfacial dilational rheology measurements. The combination of the interfacial characterization with studies on the association phenomena occurring in solution has evidenced that even the formation of kinetically trapped aggregates in the bulk occurs following similar patterns in both studied systems. These evolve following mechanisms depending of the specific chemical nature of the surfactant involved.

The equilibration of the interfacial layers formed by polyelectrolyte oppositely charged surfactants can be explained on the basis of a two-step mechanism. The first step is common to the different systems studied and is related with the diffusion-controlled incorporation of kinetically trapped aggregates at the water-vapor interfaces. Such aggregates can remain as compact aggregates at the interface, as in PDADMAC-SLMT solutions, or can undergo dissociation and spreading along the 
interface due to Marangoni flows, as in PDADMAC-SLES solutions. These different mechanisms result from differences in the hydrophobicity of the formed aggregates and the possibility to establish a cohesion interaction, such as a hydrogen bond, with the interface. On the basis of the obtained results, it can be concluded that there are no general laws governing the equilibration of the interfacial layers formed by the adsorption of polyelectrolyte-surfactant solutions at the fluid interface, with the process being primarily controlled by the specific nature of the chemical compounds involved and the interactions involved in the equilibration of the interface. This study contributes to the understanding of the fundamental basis describing the interfacial behavior of polyelectrolyte-surfactant solutions in conditions similar to that used in industrial application. Thus, the obtained result can help to exploit the interfacial behavior of these systems in technologically relevant conditions.

Author Contributions: Conceptualization, L.F.-P., A.A., and S.L.; Methodology, E.G., L.F.-P., A.A., and S.L.; Software, E.G.; Validation, E.G., F.O. and R.G.R.; Formal Analysis, E.G.; Investigation, E.G., L.F.P., A.A., S.L., F.O., and R.G.R.; Resources, R.G.R. and F.O.; Data Curation, E.G.; Writing-Original Draft Preparation, E.G.; Writing-Review and Editing, E.G., F.O. and R.G.R.; Visualization, E.G.; Supervision, E.G., F.O. and R.G.R.; Project Administration, R.G.R.; Funding Acquisition, R.G.R. and F.O.

Funding: This work was funded by MINECO under grant CTQ-2016-78895-R.

Acknowledgments: The CAI of spectroscopy from Universidad Complutense de Madrid is acknowledged for granting access to its facilities.

Conflicts of Interest: The authors declare no conflict of interest. The funders had no role in the design of the study; in the collection, analyses, or interpretation of data; in the writing of the manuscript, or in the decision to publish the results.

\section{References}

1. Llamas, S.; Guzmán, E.; Ortega, F.; Baghdadli, N.; Cazeneuve, C.; Rubio, R.G.; Luengo, G.S. Adsorption of polyelectrolytes and polyelectrolytes-surfactant mixtures at surfaces: A physico-chemical approach to a cosmetic challenge. Adv. Colloid Interface Sci. 2015, 222, 461-487. [CrossRef] [PubMed]

2. Goddard, E.D.; Ananthapadmanabhan, K.P. Application of Polymer-Surfactant Systems; Marcel Dekker, Inc.: New York, NY, USA, 1998.

3. Bain, C.D.; Claesson, P.M.; Langevin, D.; Meszaros, R.; Nylander, T.; Stubenrauch, C.; Titmuss, S.; von Klitzing, R. Complexes of surfactants with oppositely charged polymers at surfaces and in bulk. Adv. Colloid Interface Sci. 2010, 155, 32-49. [CrossRef] [PubMed]

4. Khan, N.; Brettmann, B. Intermolecular interactions in polyelectrolyte and surfactant complexes in solution. Polymers 2019, 11, 51. [CrossRef] [PubMed]

5. Gradzielski, M.; Hoffmann, I. Polyelectrolyte-surfactant complexes (PESCs) composed of oppositely charged components. Curr. Opin. Colloid Interface Sci. 2018, 35, 124-141. [CrossRef]

6. Guzmán, E.; Llamas, S.; Maestro, A.; Fernández-Peña, L.; Akanno, A.; Miller, R.; Ortega, F.; Rubio, R.G. Polymer-surfactant systems in bulk and at fluid interfaces. Adv. Colloid Interface Sci. 2016, 233, 38-64. [CrossRef] [PubMed]

7. Varga, I.; Campbell, R.A. General physical description of the behavior of oppositely charged polyelectrolyte/surfactant mixtures at the air/water interface. Langmuir 2017, 33, 5915-5924. [CrossRef] [PubMed]

8. Nylander, T.; Samoshina, Y.; Lindman, B. Formation of polyelectrolyte-surfactant complexes on surfaces. Adv. Colloid Interface Sci. 2006, 123-126, 105-123. [CrossRef]

9. Ferreira, G.A.; Loh, W. Liquid crystalline nanoparticles formed by oppositely charged surfactant-polyelectrolyte complexes. Curr. Opin. Colloid Interface Sci. 2017, 32, 11-22. [CrossRef]

10. Piculell, L.; Lindman, B. Association and segregation in aqueos polymer/polymer, polymer/surfactant, and surfactant/surfactant mixtures: Similarities and differences. Adv. Colloid Interface Sci. 1992, 41, 149-178. [CrossRef]

11. Liu, J.Y.; Wang, J.G.; Li, N.; Zhao, H.; Zhou, H.J.; Sun, P.C.; Chen, T.H. Polyelectrolyte-surfactant complex as a template for the synthesis of zeolites with intracrystalline mesopores. Langmuir 2012, 28, 8600-8607. [CrossRef] 
12. Miyake, M. Recent progress of the characterization of oppositely charged polymer/surfactant complex in dilution deposition system. Adv. Colloid Interface Sci. 2017, 239, 146-157. [CrossRef] [PubMed]

13. Szczepanowicz, K.; Bazylińska, U.; Pietkiewicz, J.; Szyk-Warszyńska, L.; Wilk, K.A.; Warszyński, P. Biocompatible long-sustained release oil-core polyelectrolyte nanocarriers: From controlling physical state and stability to biological impact. Adv.Colloid Interface Sci. 2015, 222, 678-691. [CrossRef] [PubMed]

14. Picullel, L. Understanding and exploiting the phase behavior of oppositely charged polymer and surfactant in water. Langmuir 2013, 29, 10313-10329. [CrossRef] [PubMed]

15. Goddard, E.D. Polymer/surfactant interaction: Interfacial aspects. J. Colloid Interface Sci. 2002, 256, $228-235$. [CrossRef]

16. Llamas, S.; Guzmán, E.; Akanno, A.; Fernández-Peña, L.; Ortega, F.; Campbell, R.A.; Miller, R.; Rubio, R.G. Study of the liquid/vapor interfacial properties of concentrated polyelectrolyte-surfactant mixtures using surface tensiometry and neutron reflectometry: Equilibrium, adsorption kinetics, and dilational rheology. J. Phys. Chem. C 2018, 122, 4419-4427. [CrossRef]

17. Campbell, R.A.; Arteta, M.Y.; Angus-Smyth, A.; Nylander, T.; Varga, I. Effects of bulk colloidal stability on adsorption layers of poly(diallyldimethylammonium chloride)/sodium dodecyl sulfate at the air/water interface studied by neutron reflectometry. J. Phys. Chem. B 2011, 115, 15202-15213. [CrossRef] [PubMed]

18. Campbell, R.A.; Arteta, M.Y.; Angus-Smyth, A.; Nylander, T.; Varga, I. Multilayers at interfaces of an oppositely charged polyelectrolyte/surfactant system resulting from the transport of bulk aggregates under gravity. J. Phys. Chem B 2012, 116, 7981-7990. [CrossRef] [PubMed]

19. Campbell, R.A.; Arteta, M.Y.; Angus-Smyth, A.; Nylander, T.; Noskov, B.A.; Varga, I. Direct impact of non-equilibrium aggregates on the structure and morphology of pdadmac/SDS layers at the air/water interface. Langmuir 2014, 30, 8664-8774. [CrossRef] [PubMed]

20. Mészáros, R.; Thompson, L.; Bos, M.; Varga, I.; Gilányi, T. Interaction of sodium dodecyl sulfate with polyethyleneiminie: surfactant-induced polymer solution colloid dispersion transition. Langmuir 2003, 19, 609-615. [CrossRef]

21. Mezei, A.; Pojják, K.; Mészaros, R. Nonequilibrium features of the association between poly(vinylamine) and sodium dodecyl sulfate: The validity of the colloid dispersion concept. J. Phys. Chem B 2008, 112, 9693-9699. [CrossRef] [PubMed]

22. Pojják, K.; Bertalanits, E.; Mészáros, R. Effect of salt on the equilibrium and nonequilibrium features of polyelectrolyte/surfactant association. Langmuir 2011, 27, 9139-9147. [CrossRef] [PubMed]

23. Bodnár, K.; Fegyver, E.; Nagy, M.; Mészáros, R. Impact of polyelectrolyte chemistry on the thermodynamic stability of oppositely charged macromolecules/surfactant mixtures. Langmuir 2016, 32, 1259-1268. [CrossRef] [PubMed]

24. Goddard, E.D.; Hannan, R.B. Cationic polymer/anionic surfactant interactions. J. Colloid Interface Sci. 1976, 55, 73-79. [CrossRef]

25. Bergeron, V.; Langevin, D.; Asnacios, A. Thin-film forces in foam films containing anionic polyelectrolyte and charged surfactants. Langmuir 1996, 12, 1550-1556. [CrossRef]

26. Bhattacharyya, A.; Monroy, F.; Langevin, D.; Argillier, J.-F. Surface rheology and foam stability of mixed surfactant-polyelectrolyte solutions. Langmuir 2000, 16, 8727-8732. [CrossRef]

27. Stubenrauch, C.; Albouy, P.-A.; von Klitzing, R.; Langevin, D. Polymer/surfactant complexes at the water/air interface: A surface tension and x-ray reflectivity study. Langmuir 2000, 16, 3206-3213. [CrossRef]

28. Braun, L.; Uhlig, M.; von Klitzing, R.; Campbell, R.A. Polymers and surfactants at fluid interfaces studied with specular neutron reflectometry. Adv. Colloid Interface Sci. 2017, 247, 130-148. [CrossRef]

29. Lu, J.R.; Thomas, R.K.; Penfold, J. Surfactant layers at the air/water interface: Structure and composition. Adv. Colloid Interface Sci. 2000, 84, 143-304. [CrossRef]

30. Narayanan, T.; Wacklin, H.; Konovalov, O.; Lund, R. Recent applications of synchrotron radiation and neutrons in the study of soft matter. Crystallography Rev. 2017, 23, 160-226. [CrossRef]

31. Staples, E.; Tucker, I.; Penfold, J.; Warren, N.; Thomas, R.K.; Taylor, D.J.F. Organization of polymer-surfactant mixtures at the air-water interface: sodium dodecyl sulfate and poly(dimethyldiallylammonium chloride). Langmuir 2002, 18, 5147-5153. [CrossRef]

32. Penfold, J.; Tucker, I.; Thomas, R.K.; Zhang, J. Adsorption of polyelectrolyte/surfactant mixtures at the air-solution interface:poly(ethyleneimine)/sodium dodecyl sulfate. Langmuir 2005, 21, 10061-10073. [CrossRef] [PubMed] 
33. Penfold, J.; Thomas, R.K.; Taylor, D.J.F. Polyelectrolyte/surfactant mixtures at the air-solution interface. Curr. Opin. Colloid Interface Sci. 2006, 11, 337-344. [CrossRef]

34. Penfold, J.; Tucker, I.; Thomas, R.K.; Taylor, D.J.F.; Zhang, X.L.; Bell, C.; Breward, C.; Howell, P. The interaction between sodium alkyl sulfate surfactants and the oppositely charged polyelectrolyte, polyDMDAAC, at the air-water interface: The role of alkyl chain length and electrolyte and comparison with theoretical predictions. Langmuir 2007, 23, 3128-3136. [CrossRef] [PubMed]

35. Thomas, R.K.; Penfold, J. Thermodynamics of the air-water interface of mixtures of surfactants with polyelectrolytes, oligoelectrolyte, and multivalent metal electrolytes. J. Phys. Chem B 2018, 122, 12411-12427. [CrossRef] [PubMed]

36. Bell, C.G.; Breward, C.J.W.; Howell, P.D.; Penfold, J.; Thomas, R.K. A theoretical analysis of the surface tension profiles of strongly interacting polymer-surfactant systems. J. Colloid Interface Sci. 2010, 350, 486-493. [CrossRef] [PubMed]

37. Bahramian, A.; Thomas, R.K.; Penfold, J. The adsorption behavior of ionic surfactants and their mixtures with nonionic polymers and with polyelectrolytes of opposite charge at the air-water interface. J. Phys. Chem. B 2014, 118, 2769-2783. [CrossRef] [PubMed]

38. Campbell, R.A.; Angus-Smyth, A.; Yanez-Arteta, M.; Tonigold, K.; Nylander, T.; Varga, I. New perspective on the cliff edge peak in the surface tension of oppositely charged polyelectrolyte/surfactant mixtures. J. Phys. Chem. Lett. 2010, 1, 3021-3026. [CrossRef]

39. Ábraham, A.; Campbell, R.A.; Varga, I. New method to predict the surface tension of complex synthetic and biological polyelectrolyte/surfactant mixtures. Langmuir 2013, 29, 11554-11559. [CrossRef] [PubMed]

40. Angus-Smyth, A.; Bain, C.D.; Varga, I.; Campbell, R.A. Effects of bulk aggregation on PEI-SDS monolayers at the dynamic air-liquid interface: Depletion due to precipitation versus enrichment by a convection/spreading mechanism. Soft Matter 2013, 9, 6103-6117. [CrossRef]

41. Campbell, R.A.; Tummino, A.; Noskov, B.A.; Varga, I. Polyelectrolyte/surfactant films spread from neutral aggregates. Soft Matter 2016, 12, 5304-5312. [CrossRef] [PubMed]

42. Noskov, B.A.; Loglio, G.; Miller, R. Dilational surface visco-elasticity of polyelectrolyte/surfactant solutions: Formation of heterogeneous adsorption layers. Adv. Colloid Interface Sci. 2011, 168, 179-197. [CrossRef] [PubMed]

43. Lyadinskaya, V.V.; Bykov, A.G.; Campbell, R.A.; Varga, I.; Lin, S.Y.; Loglio, G.; Miller, R.; Noskov, B.A. Dynamic surface elasticity of mixed poly(diallyldimethylammoniumchloride)/sodium dodecyl sulfate/ $\mathrm{NaCl}$ solutions. Colloids Surf. A 2014, 460, 3-10. [CrossRef]

44. Monteux, C.; Fuller, G.G.; Bergeron, V. Shear and dilational surface rheology of oppositely charged polyelectrolyte/surfactant microgels adsorbed at the air-water interface. Influence on foam stability. J. Phys. Chem. B 2004, 108, 16473-16482. [CrossRef]

45. Noskov, B.A.; Grigoriev, D.O.; Lin, S.Y.; Loglio, G.; Miller, R. Dynamic surface properties of polyelectrolyte/surfactant adsorption films at the air/water interface: Poly(diallyldimethylammonium chloride) and sodium dodecylsulfate. Langmuir 2007, 23, 9641-9651. [CrossRef] [PubMed]

46. Noskov, B.A. Dilational surface rheology of polymer and polymer/surfactant solutions. Curr. Opin. Colloids Interface Sci. 2010, 15, 229-236. [CrossRef]

47. Fauser, H.; von Klitzing, R.; Campbell, R.A. Surface adsorption of oppositely charged C14TAB-PAMPS mixtures at the air/water interface and the impact on foam film stability. J. Phys. Chem. B 2015, 119, 348-358. [CrossRef]

48. Fuller, G.G.; Vermant, J. Complex fluid-fluid interfaces: Rheology and structure. Annu. Rev. Chem. Biomol. Eng. 2012, 3, 519-543. [CrossRef]

49. Regismond, S.T.A.; Winnik, F.M.; Goddard, E.D. Surface viscoelasticity in mixed polycation anionic surfactant systems studied by a simple test. Colloids Surf. A 1996, 119, 221-228. [CrossRef]

50. Llamas, S.; Fernández-Peña, L.; Akanno, A.; Guzmán, E.; Ortega, V.; Ortega, F.; Csaky, A.G.; Campbell, R.A.; Rubio, R.G. Towards understanding the behavior of polyelectrolyte-Surfactant mixtures at the water/vapor interface closer to technologically-relevant conditions. Phys. Chem. Chem. Phys. 2018, 20, 1395-1407. [CrossRef]

51. Llamas, S.; Guzmán, E.; Baghdadli, N.; Ortega, F.; Cazeneuve, C.; Rubio, R.G.; Luengo, G.S. Adsorption of poly(diallyldimethylammonium chloride)-sodium methyl-cocoyl-taurate complexes onto solid surfaces. Colloids Surf. A 2016, 505, 150-157. [CrossRef] 
52. Mendoza, A.J.; Guzmán, E.; Martínez-Pedrero, F.; Ritacco, H.; Rubio, R.G.; Ortega, F.; Starov, V.M.; Miller, R. Particle laden fluid interfaces: Dynamics and interfacial rheology. Adv.Colloid Interface Sci. 2014, 206, 303-319. [CrossRef] [PubMed]

53. Goddard, E.D.; Gruber, J.V. Principles of Polymer Science and Technology in Cosmetics and Personal Care; Marcel Dekker, Inc.: Basel, Switzerland, 1999.

54. Mezei, A.; Mezaros, R. Novel method for the estimation of the binding isotherms of ionic surfactants on oppositely charged polyelectrolytes. Langmuir 2006, 22, 7148-7151. [CrossRef] [PubMed]

55. Naderi, A.; Claesson, P.M.; Bergström, M.; Dedinaite, A. Trapped non-equilibrium states in aqueous solutions of oppositely charged polyelectrolytes and surfactants: Effects of mixing protocol and salt concentration. Colloids Surf. A 2005, 253, 83-93. [CrossRef]

56. Akanno, A. Bulk and Surface Properties of Polyelectrolyte Surfactant Mixtures; Universidad Complutense de Madrid: Madrid, Spain, 2018.

57. Mezei, A.; Mészáros, R.; Varga, I.; Gilanyi, T. Effect of mixing on the formation of complexes of hyperbranched cationic polyelectrolytes and anionic surfactants. Langmuir 2007, 23, 4237-4247. [CrossRef] [PubMed]

58. Noskov, B.A.; Bilibin, A.Y.; Lezov, A.V.; Loglio, G.; Filippov, S.K.; Zorin, I.M.; Miller, R. Dynamic surface elasticity of polyelectrolyte solutions. Colloids Surf. A 2007, 298, 115-122. [CrossRef]

59. Tummino, A.; Toscano, J.; Sebastiani, F.; Noskov, B.A.; Varga, I.; Campbell, R.A. Effects of aggregate charge and subphase ionic strength on the properties of spread polyelectrolyte/surfactant films at the air/water interface under static and dynamic conditions. Langmuir 2018, 34, 2312-2323. [CrossRef]

60. Llamas, S. Estudio de Interfases de Interés en Cosmética; Universidad Complutense de Madrid: Madrid, Spain, 2014.

61. Erickson, J.S.; Sundaram, S.; Stebe, K.J. Evidence that the induction time in the surface pressure evolution of lysozyme solutions is caused by a surface phase transition. Langmuir 2000, 16, 5072-5078. [CrossRef]

62. Schramm, L.L. Emulsions, Foams, Suspensions, and Aerosols; Wiley-VCH Verlag GmbH \& Co.: Weinheim, Germany, 2014.

63. Langevin, D. Aqueous foams: A field of investigation at the frontier between chemistry and physics. ChemPhysChem 2008, 9, 510-522. [CrossRef]

64. Liggieri, L.; Santini, E.; Guzmán, E.; Maestro, A.; Ravera, F. Wide-frequency dilational rheology investigation of mixed silica nanoparticle-CTAB interfacial layers. Soft Matter 2011, 7, 6699-7709. [CrossRef]

65. Ravera, F.; Ferrari, M.; Santini, E.; Liggieri, L. Influence of surface processes on the dilational visco-elasticity of surfactant solutions. Adv. Colloid Interface Sci. 2005, 117, 75-100. [CrossRef] 